\title{
Perfil de Indicadores da Atenção Primária à Saúde no Estado de São Paulo: retrospectiva de 10 anos
}

\section{Primary Health Care Indicators' Profile in the State of São Paulo: 10-year retrospective}

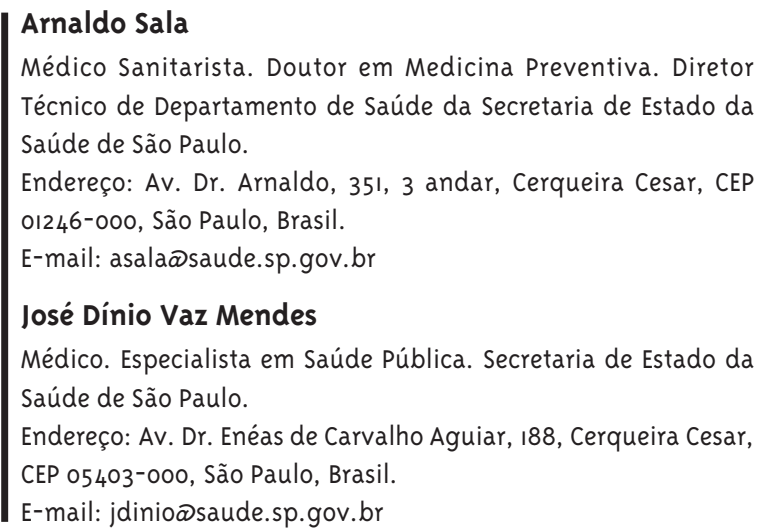

Médico. Especialista em Saúde Pública. Secretaria de Estado da Saúde de São Paulo.

Endereço: Av. Dr. Enéas de Carvalho Aguiar, 188, Cerqueira Cesar, CEP 05403-000, São Paulo, Brasil.

E-mail: jdinioœsaude.sp.gov.br

\section{Resumo}

Há mais de 20 anos da criação do Sistema Único de Saúde (SUS) e após longo período de incremento da atenção primária à saúde (APS) no Estado de São Paulo, Brasil, uma das mais importantes tarefas é avaliar seus processos e resultados. 0 objetivo deste estudo é analisar o perfil de indicadores relacionados à APS no Estado de São Paulo, de 2000 a 2009. Foram selecionados 14 indicadores de estrutura, desempenho do SUS e de condições de saúde, relacionados à APS. Esses indicadores foram analisados considerando o Estado de São Paulo como um todo e por grupos de municípios segundo portes populacionais (pequeno: menos de 20 mil habitantes; médio: entre 20 e 100 mil; grande: acima de 100 mil) e "riqueza" (alta; baixa). Quase todos dos 14 indicadores tiveram incremento favorável no período. Os indicadores de estrutura da APS apresentaram incremento, sendo que os grupos dos pequenos municípios apresentaram melhores níveis no período. Os indicadores de desempenho mostraram diferenças entre grupos de municípios. 0 grupo dos grandes municípios obteve taxas mais baixas de internações nas condições sensíveis à APS. No entanto, esse grupo obteve as piores taxas de exames citopatológicos de colo uterino. 0 grupo dos municípios pequenos e ricos apresentou melhor evolução dos indicadores de condições de saúde. 0 grupo dos grandes municípios apresentou as maiores taxas de mortalidade por câncer de colo uterino e por doença do aparelho circulatório. Concluindo, verificou-se uma clara melhora na APS, em termos de estrutura, desempenho da atenção e do estado de saúde.

Palavras-chave: Atenção primária à saúde; Avaliação; Sistema Único de Saúde; Indicadores de saúde. 


\section{Abstract}

More than 20 years after the creation of Sistema Unico de Saúde (SUS - Brazil's National Health System) and after a long period of improvements in primary health care (PHC) in the State of São Paulo, Brazil, one of the most important tasks is to evaluate their processes and their health outcomes. The aim of this study is to analyze the profile of indicators related to PHC in the State of São Paulo during the last decade (2000 to 2009). Fourteen indicators related to PHC structure, SUS performance and to health conditions were selected. These indicators were analyzed considering the entire State of São Paulo and by groups of municipalities defined by their population (small: less than 20 thousand inhabitants; medium: between 20 and 100 thousand inhabitants; large: more than 100 thousand inhabitants) and by their wealth (low; high). Almost all the 14 selected indicators improved along the period. The PHC structure-related indicators had a global improvement, and the groups of small municipalities showed better levels along the entire period. The SUS performance-related indicators showed differences among groups of municipalities. The group of large municipalities obtained lower hospitalization rates in conditions preventable by PHC. Nevertheless, this same group had the lowest rates of pap smear tests. The group of small and high wealth municipalities presented better trends in health condition indicators. The group of large municipalities presented the highest rates of mortality caused by cervical cancer and cardiovascular disease. In conclusion, there is a clear improvement in PHC in terms of structure, health care performance and health conditions.

Keywords: Primary Health Care; Evaluation; National Health System; Health Indicators.

\section{Introdução}

Pouco mais de 20 anos após a criação do Sistema Único de Saúde (SUS) e de cerca de 15 anos em que o processo de descentralização e municipalização trouxe para a esfera municipal a responsabilidade pela operação das ações relacionadas à Atenção Primária à Saúde (APS), cabe uma análise da evolução das modificações obtidas no estado de saúde da população e no desempenho das ações.

No Estado de São Paulo, verifica-se uma longa tradição na estruturação e no desenvolvimento de ações que compõem a APS. Na década de 1970 operou-se a estruturação de um modelo de organização das ações baseada na Programação em Saúde e na estruturação de uma extensa rede de Centros de Saúde operada pela Secretaria de Estado da Saúde, lócus privilegiado para a pretendida integração entre as ações de caráter coletivo e as ações dirigidas ao controle de saúde dos indivíduos (Nemes, 1990; Gonçalves, 1994). Na década seguinte, esse modelo já se apresentava em crise, tensionado pela necessidade de responder à crescente pressão de demanda por atenção médica individual.

A municipalização da APS, em São Paulo, ocorre nesse cenário de existência de uma rede de Centros de Saúde transferida para a gestão municipal, que se soma a uma rede municipal de Unidades Básicas de Saúde (UBS) em processo de expansão, sobretudo nas grandes cidades.

A implantação do Programa de Saúde da Família (PSF) no Estado de São Paulo, em 1998, e a expansão de sua cobertura nos anos seguintes encontram, assim, uma rede de unidades de APS já instalada e operando segundo um modelo diferente do proposto pelo PSF.

No Brasil, o processo de implantação do PSF ocorreu de modo mais acelerado e com muita aceitação nos municípios pequenos e médios (Bodstein e col., 2006). Desse modo, o Ministério da Saúde traçou política de estímulo à expansão do Programa nos municípios com população acima de 100 mil habitantes, por meio do Projeto de Expansão e Consolidação do Programa de Saúde da Família (Proesf).

Uma das tarefas a que se propôs o Proesf foi a de implementar os processos de avaliação e monitoramento da Atenção Básica. Os Estudos de Linha 
de Base do Proesf, realizados em vários estados brasileiros, buscaram, com diferentes abordagens metodológicas, tratar do desempenho da APS em municípios com população acima de 100 mil habitantes. Facchini e colaboradores (2006) avaliaram o desempenho da APS em estados da região Nordeste e da região Sul comparando o modelo do PSF com o da atenção básica tradicional, por meio de coleta de dados primários, analisando as dimensões político-institucional, de organização da atenção e de desempenho das ações na unidade básica. Nesta última dimensão, analisaram as ações de promoção à saúde e os perfis de morbidade, procurando comparar diferenças de desempenho entre as unidades de PSF e as tradicionais.

Em São Paulo, Ibañez e colaboradores (2006) também avaliaram o desempenho da APS a partir da coleta de dados primários, buscando considerar as categorias de acesso, porta de entrada, vínculo, integralidade, coordenação, enfoque familiar, orientação para a comunidade e formação profissional. Foram analisados resultados segundo clusters de municípios com mais de 100 mil habitantes, definidos a partir de indicadores sociais e de complexidade da estrutura de serviços de saúde, com a exclusão do município de São Paulo, por possuir características muito diferentes dos demais.

Elias e colaboradores (2006) compararam, no município de São Paulo, a atenção das unidades básicas tradicionais e do Programa de Saúde da Família por meio de aplicação de questionário avaliando a opinião de usuários e profissionais dos serviços sobre diversas dimensões da atenção primária.

Viana e colaboradores (2006) também citaram resultados de Estudo de Linha de Base no estado de São Paulo, apresentando análise a partir dos mesmos clusters de municípios. A partir de dados secundários relativos à produção ambulatorial básica, consultas e exames especializados e cobertura do PSF, também agrupam os municípios perfis de produção ambulatorial. As avaliações de efetividade, eficácia, sustentabilidade e governabilidade utilizaram fontes de dados primários.

Outra abordagem envolvendo ainda os Estudos de Linha de Base do Proesf procurou mensurar o impacto do PSF em indicadores relacionados à saúde da criança, em municípios do Nordeste brasileiro
(Roncalli e Lima, 20o6). Envolveram indicadores de cobertura vacinal, internações por diarreia e por doença respiratória, mortalidade infantil e baixo peso ao nascer.

No estado do Rio de Janeiro, a pesquisa na perspectiva dos Estudos de Linha de Base considerou um inquérito domiciliar utilizando questionário inspirado na Pesquisa Mundial de Saúde proposta pela Organização Mundial de Saúde (OMS) (Szwarcwald e col., 2006). Esse estudo envolveu indicadores de atendimento pré-natal e assistência ao parto, saúde infantil, prevenção de câncer de colo uterino e mama, assistência a idosos, assistência bucal, fatores de risco (obesidade, fumo, atividade física e dieta) e desempenho do sistema de saúde. Esses indicadores focam, sobretudo, questões relacionadas às ações de promoção e controle de saúde decorrentes do contato com os serviços de APS.

A maior parte dos estudos de avaliação da APS acaba por abordar seu desempenho (em diferentes aspectos conceituais e operacionais), tomado a partir da realização de ações em saúde, relacionando-o ao contexto demográfico e sociopolítico dos municípios. Em geral procura diferenciar a atenção prestada em UBS tradicionais da estratégia do PSF, adotada pelo Ministério da Saúde para a organização da APS no país.

No entanto, poucos estudos publicados até o momento realizaram a mensuração de impacto epidemiológico atribuível às ações da APS. Mesmo nesses casos, buscou-se principalmente diferenciar os resultados do PSF em relação ao modelo tradicional das UBS mais do que verificar o impacto dessa forma de atenção nos perfis de saúde de suas regiões e municípios.

O acompanhamento das ações em saúde relacionadas à APS, bem como dos seus resultados sobre a saúde das populações, tem sido preocupação de gestores do SUS. Os processos de pactuação de indicadores e metas entre as três esferas de governo que compõem o SUS são decorrentes não só da necessidade de pactuar metas (que em última análise são parte dos instrumentos de planejamento do SUS), mas da necessidade de acompanhar a realização das ações e também de mensurar os efeitos na saúde das pessoas. A partir de 2006, com o estabelecimento do Pacto pela Saúde, a pactuação de indicadores envol- 
ve um conjunto de 54 indicadores que recobrem as prioridades do Pacto pela Vida e do Pacto de Gestão (Brasil, 2010).

A Secretaria de Estado da Saúde de São Paulo (SES-SP), nessa vertente de preocupações, vem publicando anualmente uma Matriz de Indicadores de Saúde ${ }^{1}$, < cujos indicadores são reunidos em quatro categorias: demográficos e socioeconômicos; condições de vida e saúde; rede de serviços; financiamento. Assim, essa Matriz permite não só o acesso mais fácil a esse tipo de dados, mas pretende ser instrumento auxiliar nos processos de planejamento regional de saúde. As versões anuais dessa Matriz de Indicadores permitem, ainda, ao longo do tempo, visualizar possíveis modificações no contexto socioeconômico, no desempenho do sistema de saúde (rede de serviços) ou nos indicadores epidemiológicos.

Como salientado por Barata e colaboradores (2010), existem diversos problemas assistenciais e estruturais na atenção básica no estado de São Paulo. Em levantamento realizado pela equipe técnica da Secretaria de Estado de Saúde em um conjunto de municípios com menos de 100 mil habitantes, visitando-se mais de 1.500 unidades, constatou-se que $34 \%$ das unidades qualificadas como PSF não faziam vacinação e 18\% não tinham consultório ginecológico. Entre outras questões são também apontadas a inexistência de mecanismos e padrões definidos de referência e contrarreferência entre a atenção básica e outros níveis de saúde, o excesso de internações evitáveis, o atraso na detecção e no diagnóstico precoce e no início de tratamento de doenças como hipertensão arterial e diabetes, a baixa qualidade do acompanhamento pré-natal, tornando fundamental o melhor conhecimento e a avaliação da situação da atenção básica e seus resultados, permitindo assim a elaboração de novas medidas para seu aperfeiçoamento.

Uma vez que ocorreram modificações significativas na estrutura e operação da APS, com a municipalização da responsabilidade da gestão e operação da APS e a expansão do PSF, este estudo tem como objetivo analisar o comportamento de alguns indicadores selecionados pertinentes à APS, no estado de São Paulo, abrangendo os últimos 10 anos (2000 a 2009). Considera-se a hipótese de que municípios com diferentes contextos de população e desenvolvimento social possam obter respostas diferentes no desenvolvimento da APS, em seu desempenho e nos perfis de saúde resultantes.

\section{Métodos}

Estudo descritivo longitudinal envolvendo os 645 municípios que compõem o estado de São Paulo, utilizando-se 14 indicadores relacionados à APS.

\section{Seleção dos indicadores da APS}

Foram selecionados alguns indicadores que pudessem descrever a situação da APS no estado de São Paulo, considerando elementos da estrutura e desempenho, bem como o estado de saúde da população, nas situações em que seria possível estabelecer relações entre ações da APS e modificações no perfil de saúde. Para esses indicadores selecionados, foram definidos os valores para o conjunto do Estado e por grupos de município para os anos de 2000 a 2009. A fonte de dados para o cálculo desses indicadores foram os sistemas de informação do SUS, a saber: o Sistema de Informação Ambulatorial (SIA), o Sistema de Informação Hospitalar (SIH), o Cadastro Nacional de Estabelecimentos de Saúde (CNES), o Sistema de Informações de Mortalidade (SIM), o Sistema de Informações de Nascidos Vivos (Sinasc) e o Sistema de Informações do Câncer do Colo do Útero (Siscolo). A estimativa populacional é do Instituto Brasileiro de Geografia e Estatística (IBGE), disponibilizada no site do Departamento de Informática do SUS (Datasus) do Ministério da Saúde. ${ }^{2}$

\section{Indicadores de estrutura da APS}

1. Cobertura do PSF.

2. Consultas médicas básicas por habitante, por ano (utilizado como indicador da oferta de atenção básica nos municípios em qualquer dos modelos de APS, PSF ou UBS tradicional).

\footnotetext{
1 Disponível em: <http://www.saude.sp.gov.br/content/gestor_informacoes_saude_matriz_indicadores_saude.mmp>. Acesso em: 8 fev. 2011.

2 Disponivel em: <http://www.datasus.gov.br>. acesso em: 28 fev. 2011.
} 


\section{Indicadores de desempenho da APS}

3. Percentual de nascidos vivos por cesárea.

4. Razão de exame citopatológico de colo uterino em mulheres de 25 a 59 anos (número de exames citopatológicos do colo do útero, em mulheres na faixa etária de 25 a 59 anos, por local de residência/ população feminina nessa faixa etária).

5. Percentual de nascidos vivos de mães que realizaram sete ou mais consultas de pré-natal.

6. Percentual de cura de casos novos de tuberculose pulmonar bacilífera (número de casos novos de tuberculose pulmonar bacilífera curados/total de casos novos por 100).

7. Percentual de Internações por Condições Sensíveis à Atenção Básica (ICSAB) (número de internações por ICSAB/total de internações por 100).

8. Coeficiente de internação por Acidente Vascular Cerebral (AVC) na faixa etária de 30 a 59 anos (internação por AVC - procedimentos SIH/SUS: 03.03.04.014-9 - por local de residência/população por $10 \mathrm{mil})$.

9. Coeficiente de internação por diabetes (internação por diabetes mellitus e suas complicações por local de residência/população por 10 mil).

\section{Indicadores de estado de saúde}

10. Coeficiente de mortalidade infantil.

11. Coeficiente de mortalidade neonatal.

12. Coeficiente de mortalidade pós-neonatal.

13. Coeficiente de mortalidade por câncer de colo uterino (C53-CID10).

14. Coeficiente de mortalidade por doença do aparelho circulatório na faixa etária de 30 a 59 anos (Capítulo IX-CID-10).

Para proceder à análise, foram utilizadas duas variáveis descritoras de contexto: a população estimada para o ano de 2009 pelo IBGE e o componente "riqueza" do Índice Paulista de Responsabilidade Social (IPRS) de 2008, calculado pela Fundação Sistema Estadual de Análise de Dados (Seade) com base nos dados de 2006. Essas variáveis de contexto foram categorizadas para facilitar a análise. Os municípios foram agrupados em três portes, segundo o tamanho da população: pequeno (população abaixo de 20 mil habitantes); médio (de 20 mil a 100 mil ha- bitantes); grande (acima de 100 mil habitantes). Para cada uma das categorias de tamanho da população, os municípios também foram agrupados segundo o componente "riqueza" do IPRS, tendo como limite o ponto mediano: alto (maior ou igual ao ponto mediano do componente "riqueza"); baixo (menor que o ponto mediano do componente "riqueza").

Como se trata de descrição do universo dos municípios paulistas, optou-se por não realizar nenhum teste estatístico para tratar as diferenças encontradas no comportamento dos indicadores selecionados entre os diferentes contextos de população e "riqueza".

\section{Resultados}

Os 645 municípios do estado de São Paulo apresentaram variações grandes no tamanho de suas populações, de modo que somente $11,3 \%$ possuíam população acima de 100 mil habitantes, enquanto $61,7 \%$ possuíam menos de 20 mil habitantes. Entre os pequenos municípios, 70,4\% estavam na categoria de componente de "riqueza" baixo; entre os municípios de médio porte, apenas 21,8\% estavam na categoria de componente de "riqueza" baixo. Entre os municípios com mais de 100 mil habitantes, todos estavam na categoria alto do componente de "riqueza" do IPRS (tabela 1). Percebe-se que, embora somente 73 municípios estivessem no grupo dos grandes municípios, eles somavam quase 3/4 da população do estado.

Os valores dos indicadores selecionados para os municípios paulistas foram apresentados para o conjunto do estado de São Paulo, no período de 2000 a 2009, na tabela 2. Pode-se observar que 10 desses indicadores apresentaram comportamento com tendência aproximadamente linear de acréscimo ou de redução, ao longo do período considerado. As consultas médicas básicas por habitante, a razão de exame citopatológico de colo uterino em mulheres de 25 a 59 anos, o percentual de ICSAB e o percentual de cura de tuberculose pulmonar bacilífera apresentaram evolução mais irregular, embora com valores marcadamente distintos entre os anos inicias e finais, revelando também uma tendência de mudança.

A cobertura do PSF mostrou uma tendência de ampliação da cobertura da população paulista ca- 
Tabela I - Distribuição dos municípios e da população* do estado de São Paulo segundo porte populacional e componente "riqueza" do IPRS, 2006

\begin{tabular}{|c|c|c|c|c|c|c|}
\hline \multirow[t]{3}{*}{ Porte** } & \multicolumn{4}{|c|}{ "Riqueza"*** } & \multicolumn{2}{|c|}{ Total } \\
\hline & \multicolumn{2}{|c|}{ Alto } & \multicolumn{2}{|c|}{ Baixo } & \multirow[b]{2}{*}{$\mathrm{N}$} & \multirow[b]{2}{*}{ População } \\
\hline & $\mathrm{N}$ & População & $\mathrm{N}$ & População & & \\
\hline Pequeno & 118 & 1.149 .443 & 280 & 1.939 .594 & 398 & 3.089 .037 \\
\hline Médio & 136 & 6.419 .219 & 38 & 1.177 .229 & 174 & 7.596 .448 \\
\hline Grande & 73 & 30.698 .604 & - & - & 73 & 30.698 .604 \\
\hline TOTAL & 327 & 38.267 .266 & 318 & 3.116 .823 & 645 & 41.384 .089 \\
\hline
\end{tabular}

Fonte: Datasus.

* População estimada (IBGE) para o ano de 2009.

** Porte: Pequeno $=$ menos de 20 mil habitantes; Médio $=$ de 20 mil a 100 mil habitantes; Grande $=$ mais de 100 mil habitantes.

*** "Riqueza": Alto = componente "riqueza" do IPRS maior ou igual ao percentil 50; Baixo = componente "riqueza" do IPRS menor que o percentil 50.

\section{Tabela 2 - Evolução dos indicadores selecionados no período 2000 a 2009, no estado de São Paulo}

\begin{tabular}{|c|c|c|c|c|c|c|c|c|c|c|}
\hline Indicador & 2000 & 2001 & 2002 & 2003 & 2004 & 2005 & 2006 & 2007 & 2008 & 2009 \\
\hline Percentual de cobertura do PSF & 6,31 & 10,10 & 13,94 & 16,88 & 19,22 & 21,10 & 24,32 & 26,00 & 27,68 & 27,96 \\
\hline $\begin{array}{l}\text { Consultas Médicas básicas por } \\
\text { habitante }\end{array}$ & 1,52 & 1,60 & 1,67 & 1,67 & 1,65 & 1,66 & 1,64 & 1,62 & ।,73 & 1,70 \\
\hline Percentual de parto cesáreo & 47,57 & 48,64 & 49,71 & 50,60 & 52,32 & 53,78 & 54,60 & 55,24 & 56,65 & 57,54 \\
\hline $\begin{array}{l}\text { Razão de exame citopatológico } \\
\text { de colo uterino }\end{array}$ & 0,03 & 0,03 & 0,03 & 0,02 & 0,01 & 0,00 & 0,16 & 0,14 & 0,17 & 0,17 \\
\hline $\begin{array}{l}\text { Porcentagem de nascidos vivos } \\
\text { com sete ou + consultas de pré-natal }\end{array}$ & 53,79 & 58,13 & 62,76 & 66,42 & 71,04 & 73,39 & 73,76 & 74,67 & 76,09 & 76,06 \\
\hline Percentual de cura de TBC & 72,10 & 74,33 & 75,94 & 78,77 & 79,60 & 76,54 & 79,42 & 78,02 & 79,58 & 77,88 \\
\hline Percentual de ICSAB & 18,41 & 18,72 & 18,63 & 19,37 & 19,03 & 18,37 & 18,05 & 17,43 & 15,95 & 16,06 \\
\hline $\begin{array}{l}\text { Coef. de internação por AVC } \\
(30 \text { a } 59 \text { anos) }\end{array}$ & 7,17 & 7,24 & 7,32 & 7,20 & 7,04 & 6,98 & 6,37 & 6,00 & 5,79 & 6,22 \\
\hline Coef. de internação por diabetes & 7,57 & 7,38 & 6,92 & 6,32 & 6,19 & 5,85 & 5,41 & 5,18 & 5,45 & 5,55 \\
\hline Coef. de mortalidade infantil & 16,97 & 16,07 & 15,04 & 14,85 & 14,25 & 13,44 & 13,28 & 13,07 & 12,56 & 12,48 \\
\hline Coef. de mortalidade neonatal & 11,45 & 10,92 & 10,45 & 10,06 & 9,75 & 9,19 & 9,01 & 8,87 & 8,59 & 8,68 \\
\hline Coef. de mortalidade pós-neonatal & 5,53 & 5,15 & 4,59 & 4,79 & 4,50 & 4,26 & 4,27 & 4,20 & 3,97 & 3,81 \\
\hline $\begin{array}{l}\text { Coef. de mortalidade por câncer } \\
\text { de colo uterino }\end{array}$ & 4,82 & 4,58 & 4,08 & 4,12 & 4,12 & 3,89 & 3,74 & 3,78 & 3,84 & 3,87 \\
\hline $\begin{array}{l}\text { Coef. de mortalidade por DAC } \\
(30 \text { a } 59 \text { anos) }\end{array}$ & 129,00 & 123,25 & 118,84 & 120,95 & 119,38 & 111,05 & III, 29 & 102,38 & 102,45 & 99,94 \\
\hline
\end{tabular}

Fonte: SAI-SUS; SIH-SUS; NNES; SIM; Sisnac; Siscolo.

dastrada mais intensa nos anos iniciais e tendendo a uma estabilização nos últimos três anos.

A proporção de consultas médicas básicas por habitante apresentou um crescimento pouco expressivo nos anos iniciais, com período de estabilização até 2007 e um incremento abrupto para 2008 e 2009, que pode ser decorrente de erro na base de dados do Sistema de Informação Ambulatorial do SUS, em que vários municípios apareceram nos dois anos com números muito acima da média verificada nos anos anteriores. Mesmo após a depuração das inconsistências mais evidentes, os valores ainda permaneceram elevados. 
Entre os indicadores de desempenho da APS, o percentual de nascidos vivos de parto cesáreo apresentou crescimento contínuo ao longo de todo o período, passando de $48 \%$ para $58 \%$ nos 10 anos considerados.

A razão de exame citopatológico de colo uterino em mulheres de 25 a 59 anos apresentou patamares muito baixos até 2005, com um aumento significativo em 2006, com valores que se mantivera estáveis até 2009.

O percentual de nascidos vivos de mães com sete ou mais consultas de pré-natal apresentou incremento contínuo ao longo do período estudado, tendendo a uma estabilização nos dois últimos anos com valores de $76 \%$.

0 percentual de cura entre os casos novos de tuberculose pulmonar bacilífera apresentou um pequeno aumento até 2004, mantendo-se posteriormente em níveis aproximadamente estabilizados.

$O$ percentual de ICSAB apresentou uma discreta ascensão até 2004, evoluindo nos anos seguintes com diminuição significativa.

O coeficiente de internação por AVC na faixa etária de 30 a 59 anos apresentou valores aproximadamente estáveis nos primeiros quatro anos, com redução progressiva a partir de 2005, mas com discreta piora em 2009.

0 coeficiente de internação por diabetes apresentou um declínio consistente até 2006, quando passou a ter valores aproximados em um mesmo patamar.

Entre os indicadores de mortalidade, observou-se uma tendência de declínio regular e consistente nos três indicadores relacionados aos óbitos infantis e às doenças do aparelho circulatório na faixa etária de 30 a 59 anos. Na mortalidade por câncer de colo uterino, ocorreu redução consistente até 2006 e um discreto aumento nos anos seguintes.

Para uma análise da evolução desses indicadores segundo diferentes portes populacionais de municípios e segundo o componente de "riqueza" do IPRS no período de 2000 a 2009 , foram apresentados os valores dos indicadores apenas para os anos 2000 e 2009, dado o comportamento aproximadamente linear nos valores desses indicadores. As tabelas de 3 a 5 apresentam, respectivamente, os indicadores de estrutura, de desempenho da APS e do estado de saúde, segundo grupos de municípios selecionados pelo porte populacional e "riqueza", para os anos 2000 e 2009.

$\mathrm{O}$ aumento relativo no percentual de cobertura do PSF nos 10 anos considerados foi maior nos grandes municípios. Porém essa cobertura foi muito maior nos municípios pequenos e médios do que nos grandes. Percebe-se que os municípios pequenos já apresentavam valores mais altos que os demais no ano 200o. Tanto nos municípios pequenos como nos médios, a cobertura do PSF foi maior nos municípios com indicador de "riqueza" baixo (tabela 3).

Tabela 3 - Indicadores de estrutura da APS para os anos 2000 e 2009, segundo grupos de municípios selecionados pelo porte populacional e "riqueza", no estado de São Paulo

\begin{tabular}{|c|c|c|c|c|c|}
\hline Indicador & Porte & "Riqueza" & N Município & 2000 & 2009 \\
\hline \multirow{5}{*}{ Percentual de cobertura do PSF } & \multirow[t]{2}{*}{ Pequeno } & Alto & 118 & 16,93 & 46,43 \\
\hline & & Baixo & 280 & 21,89 & 56,87 \\
\hline & \multirow[t]{2}{*}{ Médio } & Alto & 136 & 8,67 & 35,70 \\
\hline & & Baixo & 38 & 16,41 & 49,25 \\
\hline & Grande & Alto & 73 & 4,02 & 23,01 \\
\hline \multirow{5}{*}{ Consultas médicas básicas } & \multirow[t]{2}{*}{ Pequeno } & Alto & 118 & 2,79 & 3,30 \\
\hline & & Baixo & 280 & 2,45 & 2,95 \\
\hline & \multirow[t]{2}{*}{ Médio } & Alto & 136 & 1,90 & 1,99 \\
\hline & & Baixo & 38 & 1,66 & 2,25 \\
\hline & Grande & Alto & 73 & 1,33 & 1,47 \\
\hline
\end{tabular}

Fonte: CNES. 
O indicador de consulta médica básica por habitante apresentou maiores incrementos anuais nos municípios pequenos e nos médios com baixa "riqueza". Percebe-se que os pequenos municípios já apresentavam no ano 2000 valores mais elevados do que os municípios médios e os grandes (tabela 3).

Entre os indicadores de desempenho da APS, o percentual de parto cesáreo apresentou maiores incrementos entre os municípios pequenos, embora a magnitude do indicador tenha sido muito próxima nos diferentes portes de municípios. Entre os municípios de pequeno porte, aqueles classificados como baixo no componente "riqueza" do IPRS apresentaram maiores incrementos anuais dos percentuais de partos cesáreos (tabela 4).

A razão de exame citopatológico de colo uterino em mulheres de 25 a 59 anos apresentou variação positiva significativamente maior entre os municípios de pequeno porte e os de médio porte com baixa "riqueza". Vale ressaltar que, embora os três grupos possuíssem valores médios muito próximos em 200o, os municípios pequenos alcançaram valores bem maiores em 2009. Na estratificação por "riqueza”, os municípios com piores performances obtiveram os melhores resultados no período (tabela 4).

0 percentual de nascidos vivos com sete ou mais consultas de pré-natal apresentou evolução bastante favorável ao longo da década, alcançando valores semelhantes em 2009, não apresentando diferenças significativas pelo porte dos municípios, mas com melhor performance nos municípios com baixa "riqueza" (tabela 4 ).

0 percentual de cura nos casos novos de tuberculose pulmonar bacilífera apresentou incremento anual maior entre os municípios grandes, apresentando valor pior nos municípios com baixa performance de "riqueza" (pequenos ou médios). Percebese que em 2000 o percentual de cura era maior nos municípios com baixa "riqueza" e essa situação se inverteu em 2009 (tabela 4).

O percentual de ICSAB apresentou redução mais acentuada nos municípios de pequeno porte e progressivamente menos acentuada entre os de médio e grande porte populacional. As maiores reduções ocorreram no grupo de municípios com os maiores percentuais de ICSAB no ano 2000, início do período estudado. Na estratificação por "riqueza", os municípios classificados como alto apresentaram redução mais acentuada no percentual de internações evitáveis (tabela 4).

O coeficiente de internação por AVC na faixa etária de 30 a 59 anos apresentou maiores reduções entre os municípios pequenos e médios, que apresentaram os maiores coeficientes no ano 200o. Em 2009, as taxas nesses grupos chegaram a valores bastante próximos, mesmo nos diferentes estratos de "riqueza". No grupo dos grandes municípios, os valores já mais baixos permaneceram estáveis no período (tabela 4).

O coeficiente de internação por diabetes apresentou valores bem menores entre os grandes municípios. Entre os pequenos e médios municípios, aqueles classificados como baixo no componente "riqueza" do IPRS possuíam os maiores coeficientes de internação e redução menos acentuada (tabela 4).

O coeficiente de mortalidade infantil, bem como os componentes neonatal e pós-neonatal, apresentaram tendência de redução sem variações importantes entre os grupos de municípios de porte grande, médio e pequeno. Para 2009, a mortalidade infantil e a neonatal apresentaram menores coeficientes entre os municípios pequenos e classificados como alto no componente de "riqueza", seguido pelo grupo de grande porte. Na mortalidade pós-neonatal, os municípios pequenos com alto ou baixo componente de "riqueza" apresentaram os menores coeficientes (tabela 5).

O coeficiente de mortalidade por câncer de colo uterino apresentou tendência de redução mais acentuada entre os municípios médios com baixa "riqueza”. Em 2009, a taxa foi menor entre os municípios de porte médio e pequeno (tabela 5 ).

O coeficiente de mortalidade por doença do aparelho circulatório na faixa etária de 30 a 59 anos apresentou patamares mais elevados entre os grandes municípios. No entanto, todos os grupos apresentaram redução bastante significativa e semelhante dos coeficientes (tabela 5). 
Tabela 4 - Indicadores de desempenho da APS para os anos 2000 e 2009, segundo grupos de municípios selecionados pelo porte populacional e "riqueza", no estado de São Paulo

\begin{tabular}{|c|c|c|c|c|c|}
\hline Indicador & Porte & "Riqueza" & N Município & 2000 & 2009 \\
\hline \multirow[t]{5}{*}{ Percentual de parto cesáreo } & \multirow[t]{2}{*}{ Pequeno } & Alto & 118 & 52,62 & 66,07 \\
\hline & & Baixo & 280 & 43,36 & 58,93 \\
\hline & \multirow[t]{2}{*}{ Médio } & Alto & 136 & 50,04 & 62,50 \\
\hline & & Baixo & 38 & 44,55 & 56,87 \\
\hline & Grande & Alto & 73 & 47,46 & 56,27 \\
\hline \multirow{5}{*}{$\begin{array}{l}\text { Razão de exame citopatológico de colo } \\
\text { uterino }\end{array}$} & \multirow[t]{2}{*}{ Pequeno } & Alto & 118 & 0,03 & 0,21 \\
\hline & & Baixo & 280 & 0,04 & 0,24 \\
\hline & \multirow[t]{2}{*}{ Médio } & Alto & 136 & 0,03 & 0,18 \\
\hline & & Baixo & 38 & 0,02 & 0,24 \\
\hline & Grande & Alto & 73 & 0,03 & 0,15 \\
\hline \multirow{5}{*}{$\begin{array}{l}\text { Porcentagem de nascidos vivos com sete ou + } \\
\text { consultas de pré-natal }\end{array}$} & \multirow[t]{2}{*}{ Pequeno } & Alto & 118 & 61,46 & 78,25 \\
\hline & & Baixo & 280 & 51,69 & 75,80 \\
\hline & \multirow[t]{2}{*}{ Médio } & Alto & 136 & 57,85 & 76,67 \\
\hline & & Baixo & 38 & 47,46 & 76,96 \\
\hline & Grande & Alto & 73 & 53,34 & 75,86 \\
\hline \multirow[t]{5}{*}{ Percentual de cura de TBC } & \multirow[t]{2}{*}{ Pequeno } & Alto & 118 & 70,73 & 74,51 \\
\hline & & Baixo & 280 & 75,77 & 73,48 \\
\hline & \multirow[t]{2}{*}{ Médio } & Alto & 136 & 76,97 & 80,90 \\
\hline & & Baixo & 38 & 80,43 & 75,17 \\
\hline & Grande & Alto & 73 & 71,60 & 76,65 \\
\hline \multirow[t]{5}{*}{ Percentual de ICSAB } & \multirow[t]{2}{*}{ Pequeno } & Alto & 118 & 29,16 & 21,77 \\
\hline & & Baixo & 280 & 27,78 & 23,70 \\
\hline & \multirow[t]{2}{*}{ Médio } & Alto & 136 & 22,66 & 18,05 \\
\hline & & Baixo & 38 & 23,73 & 22,98 \\
\hline & Grande & Alto & 73 & 14,98 & 14,04 \\
\hline \multirow[t]{5}{*}{ Coef. de internação AVC 50 a 59 anos } & \multirow[t]{2}{*}{ Pequeno } & Alto & 118 & 11,85 & 8,46 \\
\hline & & Baixo & 280 & 12,74 & 8,00 \\
\hline & \multirow[t]{2}{*}{ Médio } & Alto & 136 & 11,27 & 7,16 \\
\hline & & Baixo & 38 & 10,47 & 8,28 \\
\hline & Grande & Alto & 73 & 5,73 & 5,76 \\
\hline \multirow[t]{5}{*}{ Coef. de internação por diabetes } & \multirow[t]{2}{*}{ Pequeno } & Alto & 118 & 11,41 & 8,30 \\
\hline & & Baixo & 280 & 12,72 & 10,77 \\
\hline & \multirow[t]{2}{*}{ Médio } & Alto & 136 & 12,13 & 7,91 \\
\hline & & Baixo & 38 & 14,10 & 13,21 \\
\hline & Grande & Alto & 73 & 5,91 & 4,33 \\
\hline
\end{tabular}

Fonte: SAI-SUS; SIH-SUS; SIM. 
Tabela 5 - Indicadores de estado de saúde para os anos 2000 e 2009, segundo grupos de municípios selecionados pelo porte populacional e "riqueza", no estado de São Paulo

\begin{tabular}{|c|c|c|c|c|c|}
\hline Indicador & Porte & "Riqueza" & N Município & 2000 & 2009 \\
\hline \multirow{5}{*}{$\begin{array}{l}\text { Coeficiente de mortalidade infantil (por mil } \\
\text { nascidos vivos) }\end{array}$} & \multirow[t]{2}{*}{ Pequeno } & Alto & 118 & 15,84 & 11,53 \\
\hline & & Baixo & 280 & 19,80 & 14,13 \\
\hline & \multirow[t]{2}{*}{ Médio } & Alto & 136 & 16,93 & 13,08 \\
\hline & & Baixo & 38 & 21,53 & 13,58 \\
\hline & Grande & Alto & 73 & 16,48 & 12,27 \\
\hline \multirow{5}{*}{$\begin{array}{l}\text { Coeficiente de mortalidade neonatal (por mil } \\
\text { nascidos vivos) }\end{array}$} & \multirow[t]{2}{*}{ Pequeno } & Alto & 118 & 11,39 & 8,17 \\
\hline & & Baixo & 280 & 12,92 & 10,59 \\
\hline & \multirow[t]{2}{*}{ Médio } & Alto & 136 & 11,64 & 9,36 \\
\hline & & Baixo & 38 & 14,34 & 9,20 \\
\hline & Grande & Alto & 73 & 11,02 & 8,44 \\
\hline \multirow{5}{*}{$\begin{array}{l}\text { Coeficiente de mortalidade pós-neonatal (por } \\
\text { mil nascidos vivos) }\end{array}$} & \multirow[t]{2}{*}{ Pequeno } & Alto & 118 & 4,46 & 3,36 \\
\hline & & Baixo & 280 & 6,87 & 3,54 \\
\hline & \multirow[t]{2}{*}{ Médio } & Alto & 136 & 5,29 & 3,72 \\
\hline & & Baixo & 38 & 7,19 & 4,38 \\
\hline & Grande & Alto & 73 & 5,46 & 3,83 \\
\hline \multirow{5}{*}{$\begin{array}{l}\text { Coef. de mortalidade por câncer de colo } \\
\text { uterino (por dez mil habitantes) }\end{array}$} & \multirow[t]{2}{*}{ Pequeno } & Alto & 118 & 4,02 & 3,35 \\
\hline & & Baixo & 280 & 4,22 & 3,37 \\
\hline & \multirow[t]{2}{*}{ Médio } & Alto & 136 & 3,98 & 3,44 \\
\hline & & Baixo & 38 & 4,44 & 2,41 \\
\hline & Grande & Alto & 73 & 5,06 & 4,05 \\
\hline \multirow{5}{*}{$\begin{array}{l}\text { Coef. de mortalidade por DAC ( } 30 \text { a } 59 \text { anos) } \\
\text { (por dez mil habitantes nas idades) }\end{array}$} & \multirow[t]{2}{*}{ Pequeno } & Alto & 118 & 109,93 & 84,14 \\
\hline & & Baixo & 280 & 119,01 & 84,21 \\
\hline & \multirow[t]{2}{*}{ Médio } & Alto & 136 & 123,70 & 93,60 \\
\hline & & Baixo & 38 & 113,98 & 85,18 \\
\hline & Grande & Alto & 73 & 130,98 & 102,52 \\
\hline
\end{tabular}

Fonte: SAI-SUS; SIM; Siscolo.

\section{Discussão}

De modo geral, os indicadores selecionados apresentaram evolução favorável no período estudado, com exceção do percentual de partos cesáreos, cujos valores apresentaram piora substancial, sobretudo entre os municípios médios e pequenos.

A estrutura da APS no estado de São Paulo sofreu modificações importantes na década estudada, com a expansão do PSF que atingiu cerca de $28 \%$ da população paulista. Essa cobertura é baixa em relação à do Brasil, que foi de 50,7\% em $2009^{3}$ Contudo esse fato não indica falta de recursos de APS para atender a população paulista (desassistência). Ele pode ser explicado por aspectos específicos do desenvolvimento da saúde pública no estado: a grande rede de UBS tradicionais implantada no estado anteriormente à implantação da estratégia do PSF e a maior cobertura do sistema de saúde suplementar entre todos os estados no Brasil. Enquanto o Brasil possuía 44,8 milhões de beneficiários da saúde su-

3 Disponível em:<http://dab.saude.gov.br/abnumeros.php\#numeros>. Acesso em: 28 fev. 2011. 
plementar ou 23,5\% da população em 2010 (Agência Nacional de Saúde Suplementar, 2010), o estado de São Paulo tinha 7,8 milhões de beneficiários (ou 43,2\%) no mesmo ano.

Além disso, conforme salientado por Oliveira e colaboradores (2010), no estado de São Paulo verifica-se associação entre o porte populacional dos municípios e a cobertura de planos de saúde privados: até 49 mil habitantes, a maior parte dos municípios apresenta cobertura de planos privados de saúde abaixo de $20 \%$ e a partir de 50 mil habitantes passa a predominar a faixa de cobertura maior ou igual a $20 \%$. Oliveira e colaboradores (2010) constatam ainda a associação entre a cobertura dos planos privados e a "riqueza" medida pelo Produto Interno Bruto (PIB) per capita municipal e observam que a relação entre a cobertura dos planos privados é inversamente proporcional à do PSF.

Portanto, os municípios de pequeno porte do estado de São Paulo, que não dispõem de cobertura significativa dos serviços da saúde suplementar, aderiram de forma mais efetiva à estratégia do PSF, apresentando efetivamente as maiores coberturas desse modelo.

O número de consultas médicas básicas por habitante/ano no estado apresentou valores mais altos nos municípios pequenos e médios, sendo os resultados verificados superiores aos preconizados nos parâmetros estabelecidos pelo Ministério da Saúde (Portaria GM/MS n. ${ }^{0} 1101$ de 12 de junho de 2002).

O comportamento desses dois indicadores da estrutura da APS permite inferir que a oferta é maior e possivelmente melhor estruturada entre os municípios pequenos e médios.

Note-se que, nos grandes municípios, o aumento da cobertura do PSF foi acompanhado da redução do número de consultas médicas básicas por habitante, o que sugere que a implantação do PSF ocorreu nesses grandes municípios mais intensamente como estratégia de substituição do modelo tradicional de APS, inspirado na Programação, e não como expansão da oferta de APS.

Contudo, como observam Bousquat e colabora- dores (2006), na capital paulista, que corresponde a $27 \%$ da população total do estado, o PSF foi implantado principalmente em distritos administrativos mais periféricos, caracterizados pela maior exclusão social, em expansão que fez com que o município de São Paulo passasse a ter o maior número absoluto de equipes de PSF do país. Segundo dados da Secretaria Municipal de Saúde de São Paulo, em outubro de 2010 existiam 1.196 equipes de saúde da família implantadas, com cobertura de 43,2\% $\%^{4}$.

Apesar disso, os grandes municípios apresentaram as mais baixas coberturas de PSF e de consultas básicas por habitante. Porém, possuíam cobertura de assistência médica suplementar muito maior que os pequenos, o que poderia estar representando uma alternativa assistencial às demandas em saúde. Este fato que deve ser levado em conta na avaliação de resultados, principalmente dos indicadores de processos, que normalmente se utilizam apenas de fontes de dados do SUS e não dispõem das informações referentes aos atendimentos prestados pela saúde suplementar.

Assim, nos municípios de maior porte, que concentravam $74 \%$ da população do estado, parte significativa dos procedimentos de APS estava, provavelmente, sendo realizada pelos serviços da saúde suplementar.

O aumento no percentual de partos cesáreos é preocupante, pois se distancia ainda mais das recomendações internacionais em relação ao tema. A elevação mais acentuada desse percentual entre os municípios pequenos e médios pode ser decorrente da estrutura assistencial hospitalar diferenciada: os municípios menores possuem maior oferta de leitos em pequenos hospitais privados, prestadores ou não do SUS. Nos grandes municípios, há uma oferta proporcionalmente maior de leitos públicos (hospitais universitários, hospitais estaduais e municipais), em que as indicações de parto cesáreo seriam mais criteriosas.

Um recente estudo de revisão acerca das taxas de cesárea aponta que maiores taxas de cesárea estão associadas a modelos assistenciais altamente medicalizados e com baixa participação de obste-

4 Disponível em: <http://www.prefeitura.sp.gov.br/cidade/secretarias/upload/saude/arquivos/esf/equipes_esf.pdf>. Acesso em: 28 fev. 2011. 
trizes. Destaca, ainda, que nos EUA, onde as taxas de parto cesáreo são elevadas, esse tipo de parto estaria também associado à conveniência médica e também à opção da própria gestante. Mesmo assim, as taxas nos EUA são bem inferiores à verificadas no Brasil, onde se apresentam em crescimento (Patah e Malik, 2011).

Entre os principais aspectos ou dimensões do PSF, conforme aponta Ibañez e colaboradores (2006), está a integralidade e a coordenação (ou integração) de serviços pelas unidades de APS. Diretrizes apontadas em documentos oficiais sobre o PSF (Brasil, 2001, 2007) salientam o papel reorientador do programa para a prática assistencial e como centro ordenador das redes de atenção à saúde do SUS em suas regiões. Contudo, o aumento da taxa de partos cesáreos para pacientes dos pequenos municípios indica deficiências na integração da APS e no desempenho de seu papel reordenador da assistência.

Com relação à razão de exames de citopatologia de colo uterino, os resultados foram muito bons para os pequenos e médios municípios com baixa "riqueza”, cujo valor de 0,24 em 2009 foi bem superior aos dos municípios grandes, indicando possível impacto positivo da APS. O valor é próximo ao parâmetro mínimo de o,3 e maior que a razão média no Brasil em 2009, que foi de o,18 exames/mulher/ano (Brasil, 2011). Deve-se notar que os valores bem mais baixos encontrados nos grandes municípios referem-se apenas aos exames realizados pelo SUS e não levam em conta aqueles realizados pelo sistema de saúde suplementar.

Apesar de o câncer de colo uterino apresentar longo período de desenvolvimento (até duas décadas) e o tempo de implantação do PSF nos municípios ser ainda insuficiente para que se esperem resultados significativos, verificou-se queda nos coeficientes de mortalidade por câncer de colo, embora ainda discreta.

Os altos percentuais de nascidos vivos de mães que realizaram sete ou mais consultas de pré-natal foram muito semelhantes nos grandes e pequenos municípios. No entanto, deve-se considerar que nos pequenos municípios, conforme apontado por Oliveira e colaboradores (2010), o acesso a consultas tem sido garantido principalmente pelo setor público, que responde com mais de $80 \%$ dos serviços ambulatoriais básicos, com predominância do PSF, como vimos anteriormente. Certamente, como observam Silveira e colaboradores (2001), o número de consultas pré-natais não garante a qualidade do cuidado, sendo necessárias avaliações quanto à adequação dos procedimentos, mas pelo menos indicam bom acesso para as gestantes na APS.

Desde o início do PSF, o controle da tuberculose esteve entre suas prioridades programáticas (Brasil, 1999). Embora o estado de São Paulo esteja obtendo percentuais de cura superiores à média nacional de $70,3 \%$, a meta de $85 \%$, recomendada pelo Programa Nacional de Controle da Tuberculose, ainda não foi atingida (Brasil, 2011). Destaca-se a evolução desfavorável obtida entre os municípios pequenos e de baixa "riqueza", em que pese o aumento importante da cobertura do PSF nesses locais.

O percentual de ICSAB apresentou redução ao longo do período estudado, com intensidade maior nos grupos de municípios com as maiores coberturas de PSF e com os maiores incrementos de consultas médicas básicas por habitante. No entanto, entre os municípios pequenos e médios, aqueles com o componente "riqueza" categorizado como baixo obtiveram reduções menos significativas.

A redução na proporção de internações por condições sensíveis à APS pode ser interpretada como melhoria nos processos e na cobertura efetiva da APS (Alfradique e col., 2009), embora esse indicador deva ser visto com cautela, pois pode sofrer interferência do modo como a estrutura assistencial estabelece em cada localidade critérios e facilidades para a internação de diferentes morbidades (Elias e Magajewski, 2008). Uma revisão sistemática da literatura internacional (Nedel e col., 2010) aponta para uma associação entre a redução nesse grupo de internações e a adoção de princípios fundamentais da APS, tais como a oferta adequada e continuidade da atenção e a presença de equipe multidisciplinar. No estado de São Paulo, sugere-se associação entre a ampliação da cobertura do PSF e redução nas internações por condições sensíveis à APS (Rehem e Egry, 2008).

As diferenças observadas nos municípios mais pobres, que tiveram redução inferior do ICSAB, independentemente do porte populacional, poderiam 
estar associadas a diversos fatores, entre eles não só uma possível fragilidade da APS, mas também uma oferta maior de leitos hospitalares por habitante. A propensão social do médico para hospitalizar pode ser maior em áreas mais pobres, uma vez que nesses locais os pacientes são mais vulneráveis a complicações médicas e a fatores ambientais, como apontam Elias e Magajewski (2008).

As internações por AVC e por diabetes, embora sejam integrantes das internações por condições sensíveis à APS, receberam destaque separado, como indicadores relacionados às ações de controle da hipertensão arterial e do diabetes. Os dois indicadores apresentaram redução ao longo de quase todo o período estudado, no estado de São Paulo, podendo traduzir uma melhora na efetividade do controle da hipertensão arterial e, sobretudo, do diabetes. Os maiores coeficientes de internação verificados entre os municípios menores, quando comparados aos grandes, precisam ser analisados com cuidado, pois entre os municípios mais populosos a participação da saúde suplementar é significativa.

Todos os componentes da mortalidade infantil se reduziram no período considerado, independentemente de porte do município ou de "riqueza", acompanhando tendência geral verificada no estado desde o final da década de 1970 (Mendes, 2009). Esses indicadores são sensíveis às condições socioeconômicas da população (condições de habitação, saneamento, nutrição e educação), além da assistência à saúde, dificultando sua interpretação quanto aos efeitos desta última. Assim, embora os municípios de baixa "riqueza" tenham taxas de mortalidade maiores, nota-se que a queda relativa foi maior nesses municípios para todos os componentes considerados.

Estudo de Cesse e colaboradores (2009), que analisou a tendência da mortalidade por Doenças do Aparelho Circulatório (DAC) nas capitais do Brasil de 1950 a 200o, refere que nos EUA e na Europa observa-se o declínio da mortalidade por esse grupo de agravos desde o final da década de 1960 devido ao comportamento de declínio das doenças isquêmicas do coração e das doenças cerebrovasculares. No Brasil, onde essas causas correspondem à primeira causa de óbito em todas as regiões do país, em ambos os sexos, os autores descrevem que apesar da oscilação dos dados, a mortalidade proporcional por DAC foi crescente até o ano de 1991 e, em seguida, apresentou uma diminuição até o final da série. Quando se analisou o risco de óbito por DAC por razões de mortalidades padronizadas, encontraram-se riscos decrescentes após o ano de 1980. Outro estudo, também abordando a mortalidade por doença do aparelho circulatório no Brasil (Curioni e col., 2009), mostra uma consistente redução na mortalidade por doença do aparelho circulatório no período de 1980 a 2003 no Brasil. Essa tendência também foi observada em cada uma das regiões brasileiras, mas apresentando diferentes taxas de redução. Tanto a doença cerebrovascular como a doença isquêmica do coração apresentaram redução, embora a primeira tenha apresentado redução mais acentuada. 0 estudo aponta, ainda, que a redução da mortalidade estaria associada a melhores índices de desenvolvimento social.

No estado de São Paulo, o coeficiente de mortalidade por DAC na faixa etária de 30 a 59 anos também apresentou tendência de queda, com o valor do indicador menor nos municípios pequenos no final do período estudado.

A grande influência dos fatores ambientais e do estilo de vida nesse tipo de evento, que incluem a urbanização, a industrialização, o sedentarismo, o tabagismo, entre outros, dificultam a avaliação das ações específicas do setor saúde. Além disso, no caso da mortalidade por DAC, a disponibilidade de serviços de referência especializados certamente tem impacto na evolução dos casos agudos. Apesar disso, a ampliação dos serviços de APS, a facilidade do acesso e a regularidade no fornecimento de medicamentos, como os anti-hipertensivos, podem ser legitimamente apontadas como mais um vetor para explicar a redução verificada.

\section{Considerações Finais}

A análise do comportamento de alguns indicadores relacionados à APS nas suas dimensões de estrutura, desempenho do sistema e situação de saúde, ao longo da década de 2000 a 2009 permitem concluir que ocorreu uma consistente melhora tanto na organização e operação da APS no estado de São Paulo quanto no perfil de saúde da população. 
Uma avaliação da gestão da APS não poderia se esgotar apenas na apreciação da evolução de sua estrutura de oferta de ações ou na verificação da adequação dos seus processos de atenção à saúde. Nesse aspecto, as séries históricas dos indicadores selecionados mostraram não só uma evidente expansão da oferta de APS, mas um aumento na qualidade das ações.

A apreciação sob o ponto de vista do impacto sobre a saúde das populações deve ser o foco último de uma avaliação da gestão. As séries temporais dos indicadores de mortalidade e morbidade hospitalar apresentados apontam consistentemente uma evolução bastante favorável. Por fim, seria necessário estabelecer relações entre a melhora da estrutura e do desempenho operacional da APS e a evolução verificada nos indicadores de saúde. Considerando o complexo cenário de determinações (sociais, econômicas, ambientais, culturais, etc.), que incidem sobre o estado de saúde de uma população, fica muito difícil mensurar a efetiva contribuição das ações em saúde na obtenção do resultado final de melhora do seu estado de saúde.

\section{Referências}

AGÊNCIA NACIONAL DE SAÚDE SUPLEMENTAR - ANS. Caderno de Informação da Saúde

Suplementar: beneficiários, operadoras e planos. Rio de Janeiro: Ministério de Saúde, Agência Nacional de Saúde Suplementar, 2010. 68 p. Disponível em: <http://www.ans.gov.br/index.php/ materiais-para-pesquisas/materiais-por-tipo-depublicacao/periodicos>. Acesso em: 28 fev. 2011.

ALFRADIQUE, M. E. et al. Internações por condições sensíveis à atenção primária: a construção da lista brasileira como ferramenta para medir o desempenho do sistema de saúde (Projeto ICSAP - Brasil). Cadernos de Saúde Pública, Rio de Janeiro, v. 25, n. 6, p. 1337-1349, jun. 2009.

BARATA, L. R. B.; MENDES, J. D. V.; ANDRADE, M. C. O gestor estadual e os desafios da atenção básica em saúde. In: CONSELHO NACIONAL DE SECRETÁRIOS DE SAÚDE. Realidades e perspectivas da gestão estadual do SUS. Brasília: Conselho Nacional de Secretários de Saúde, 2010. p. 71-79.
BODSTEIN, R. et al. Estudos de linha de base do Projeto de Expansão e Consolidação do Saúde da Família (ELB/PROESF): considerações sobre seu acompanhamento. Ciência \& Saúde Coletiva, Rio de Janeiro, v. 11, n. 3, p. 725-731, jul./set. 2006.

BOUSQUAT, A.; COHN, A.; ELIAS, P. E. Implantação do Programa de Saúde da Família e exclusão sócio-espacial no município de São Paulo - Brasil. Cadernos de Saúde Pública, Rio de janeiro, v. 22, n. 9, p. 1935-1943, set. 2006.

BRASIL. Ministério da Saúde. Pactuação Unificada de Indicadores: avaliação 2008. Brasília: Ministério da Saúde, 2010. 248 p. (Série Pactos pela Saúde, v. 14).

BRASIL. Ministério da Saúde. Manual para a organização da Atenção Básica. Brasília:

Secretaria de Assistência à Saúde, Ministério da Saúde, 1999.

BRASIL. Ministério da Saúde. Orientações acerca dos Indicadores de Monitoramento Avaliação do Pacto pela Saúde, nos Componentes pela Vida e de Gestão para o Biênio 2010 - 2011. Brasília, fev. 2011. Disponível em: <http://portalwebo4.saude. gov.br/sispacto/Instrutivo_Indicadores_2011.pdf>. Acesso em: 01 mar. 2011.

BRASIL. Ministério da Saúde. Política Nacional de Atenção Básica. 4. ed. Brasília: Secretaria de Assistência à Saúde/Ministério da Saúde, 2007. (Série E. Legislação de Saúde. Série Pactos pela Saúde 2oo6, v. 4). Disponível em: <http://dab. saude.gov.br/publicacoes.php>. Acesso em: o1 mar. 2011.

BRASIL. Ministério da Saúde. Secretaria Executiva. Programa de Saúde da Família. Brasília: Ministério da Saúde, 2001.

Disponível em: <http://www.ccs.saude.gov. br/saudebateaporta/mostravirtual/ingles/ publicacoes/psfo1.pdf>. Acesso em: 01 mar. 2011.

BRASIL. Ministério da Saúde. Portaria GM/ MS n.1.101, de 12 de junho de 2002. Disponível em: <http://pnass.datasus.gov.br/documentos/ normas/48.pdf $>$. Acesso em: 24 set. 2011. 
CESSE, E. A. P. et al. Tendência da mortalidade por doenças do aparelho circulatório no Brasil: 1950 a 2000. Arquivos Brasileiros de Cardiologia, São Paulo, v. 93, n. 5, p. 490-497, nov. 2009.

CURIONI, C. et al. The decline in mortality from circulatory diseases in Brazil. Revista Panamericana de Salud Pública, Washington, v. 25, n. 1, p. 9-15, jan. 2009.

ELIAS, E.; MAGAJEWSKI, F. A Atenção Primária à Saúde no sul de Santa Catarina: uma análise das internações por condições sensíveis à atenção ambulatorial, no período de 1999 a 2004. Revista Brasileira de Epidemiologia, São Paulo, v. 11, n. 4, p. 633-647, dez. 2008.

ELIAS, P. E. et al. Atenção Básica em Saúde: comparação entre PSF e UBS por estrato de exclusão social no município de São Paulo. Ciência \& Saúde Coletiva, Rio de Janeiro, v. 11, n. 3, p. 633641 , jul. - set. 2006.

FACCHINI, L. A. et al. Desempenho do PSF no Sul e no Nordeste do Brasil: avaliação institucional e epidemiológica da Atenção Básica à Saúde. Ciência \& Saúde Coletiva, Rio de Janeiro, v. 11, n. 3 , p. 669-681, jul./set. 2006.

GONÇALVES, R. B. M. Tecnologia e organização social das práticas de saúde: características tecnológicas do processo de trabalho na Rede Estadual de Centros de Saúde de São Paulo. São Paulo: Hucitec, 1994.

IBAÑEZ, N. et al. Avaliação do desempenho da atenção básica no estado de São Paulo. Ciência \& Saúde Coletiva, Rio de Janeiro, v. 11, n. 3, p. 683703, jul./set. 2006.

MENDES, J. D. V. A redução da mortalidade infantil no Estado de São Paulo. Saúde em dados contextualização. Boletim Epidemiológico Paulista, São Paulo, v. 6, n. 69, p. 1-11, set. 2009. Disponível em: <ftp://ftp.cve.saude.sp.gov.br/ doc_tec/outros/bol_bepa6909.pdf $>$. Acesso em: 28 fev. 2011.

NEDEL, F. B. et al. Características da atenção básica associadas ao risco de internar por condições sensíveis à atenção primária: revisão sistemática da literatura. Epidemiologia e Serviços de Saúde, Brasília, v. 19, n. 1, p. 61-75, jan./mar. 2010.
NEMES, M. I. B. Ação programática em saúde: recuperação histórica de uma política de programação. In: SCHRAIBER, L. B. (Org.). Programação em saúde hoje. São Paulo: Hucitec, 1990. p. 65-116.

OLIVEIRA, M. H. C. B.; REHEM, T. C. M. S. B.; AMARAL, T. C. L. Relação entre o público e o privado na atenção à saúde: o SUS e a saúde suplementar no estado de São Paulo. São Paulo: FUNDAP, 2010. v. 1. 117 p.

PATAH, L. E. M.; MALIK, A. M. Modelos de assistência ao parto e taxa de cesárea em diferentes países. Revista de Saúde Pública, São Paulo, v. 45, n. 1, p. 185-194, fev. 2011.

Rehem, T. C. M. S. B.; Egry, E. Y. Internações por condições sensíveis à Atenção Primária no Estado de São Paulo. Ciência \& Saúde Coletiva, São Paulo, 2009. Disponível em: <http://www. cienciaesaudecoletiva.com.br/artigos/artigo_int. php?id_artigo=2716> Acesso em: 25 fev. 2011.

RONCALLI, A. G.; LIMA, K. C. Impacto do Programa de Saúde da Família sobre os Indicadores de Saúde da criança em municípios de grande porte da região Nordeste do Brasil. Ciência \& Saúde Coletiva, Rio de Janeiro, v. 11, n. 3, p. 713724 , jul./set. 2006.

SILVEIRA, D. S.; SANTOS, I. S.; COSTA, J. S. D. Atenção pré-natal na rede básica: uma avaliação da estrutura e do processo. Cadernos de Saúde Pública, Rio de Janeiro, v. 17, n. 1, p. 131-139, jan./ fev. 2001.

SZWARCWALD, C. L.; MENDONÇA, M. H. M.; ANDRADE, C. L. T. Indicadores de atenção básica em quatro municípios do estado do Rio de Janeiro, 2005: resultados de inquérito domiciliar de base populacional. Ciência \& Saúde Coletiva, Rio de Janeiro, v. 11, n. 3, p. 643-655, jul./set. 2006.

VIANA, A. L. A. et al. Modelos de atenção básica nos grandes municípios paulistas: efetividade, eficácia, sustentabilidade e governabilidade. Ciência \& Saúde Coletiva, Rio de Janeiro, v. 11, n. 3 , p. 577-6o6, jul./set. 2006.

Recebido em: 21/03/2011

Aprovado em: 25/04/2011 